\title{
AN EXTREMAL PROPERTY OF THE BLOCH SPACE
}

\author{
LEE A. RUBEL ${ }^{1}$ AND RICHARD M. TIMONEY
}

\begin{abstract}
The Bloch space $\mathscr{B}$ is the space of functions $f$ analytic in the unit disc $D$ such that $\left|f^{\prime}(z)\right|\left(1-|z|^{2}\right)$ is bounded. It is shown that $\mathscr{B}$ is the largest Möbius-invariant linear space of analytic functions that can be equipped with a Möbius-invariant seminorm in such a way that there is at least one "decent" continuous linear functional on the space. The term "decent" has a simple and precise definition.
\end{abstract}

The space $\mathscr{B}$ of Bloch functions has been studied by many authors (see, e.g. [1]) because of its intrinsic interest and because it is the meeting place of several areas of analysis. We show here that $\mathscr{B}$ is the largest space with certain natural properties, thus providing another justification for studying it.

To be more precise, the main result of this note is that the space of Bloch functions is the largest Möbius-invariant linear space of analytic functions on the unit disc which can be equipped with a Möbius-invariant seminorm in such a way that there is at least one "decent" continuous linear functional on the space. The term "decent" is defined below. Point evaluation of the eleventh derivative is an example of a "decent" linear functional.

One simple consequence of the main result is that there are no nontrivial Möbius-invariant closed subspaces of $H(D)$, the linear space of analytic functions on the unit disc, equipped with the topology of uniform convergence on compacta. This consequence is "folklore", but the proof seems new.

Let $X$ be a Möbius-invariant linear space of analytic functions on the unit disc $D$ (i.e. $f \in X, \phi$ a Möbius transformation of $D$ implies $f \circ \phi \in X$ ). Let $p$ : $X \rightarrow[0, \infty)$ be a Möbius-invariant seminorm on $X$ (i.e. $p(f \circ \phi)=p(f)$ for each Möbius transformation $\phi$ of $D$ and every $f \in X$ ).

Definition. A nonzero linear functional $L$ on $X$ is said to be decent if

$$
|L(f)| \leqslant M \operatorname{Sup}\{|f(z)| \mid z \in K\},
$$

for all $f \in X$, for some $M>0$ and some compact subset $K$ of $D$.

Equivalently, $L \neq 0$ is decent if $L$ extends to a continuous linear functional on $H(D)$.

Notation. The Bloch seminorm $p_{\mathscr{B}}$ is defined by

$$
p_{\mathfrak{B}}(f)=\operatorname{Sup}\left\{\left|f^{\prime}(z)\right|\left(1-|z|^{2}\right)|z \in D|,\right.
$$

Presented to the Society, November 12, 1978; received by the editors June 19, 1978 and, in revised form, September 5, 1978.

AMS (MOS) subject classifications (1970). Primary 46E15; Secondary 30A74.

${ }^{1}$ The research of the first-named author was supported in part by a grant from the National Science Foundation. 
for $f$ analytic in $D$. The Bloch space, denoted $\mathscr{B}$, is the space of all analytic functions $f$ on $D$ for which $p_{\mathscr{B}}(f)$ is finite. See [1] for information on $\mathscr{B}$.

The group of Möbius transformations from $D$ onto $D$ will be denoted by $\operatorname{Aut}(D)$. Each $\phi \in \operatorname{Aut}(D)$ may be written as

for some $a \in D$ and $\lambda \in \mathbf{R}$.

$$
\phi(z)=e^{i \lambda}(z+a) /(1+\bar{a} z)
$$

The main result of this note is the following one.

THEOREM. Let $X$ be a Möbius-invariant linear space of analytic functions on the unit disc and let $p$ be a Möbius-invariant seminorm on $X$. If there exists a decent linear functional $L$ on $X$ continuous with respect to $p$, then $X \subseteq \mathscr{B}$ and there exists a constant $A>0$ such that

for all $f \in X$.

$$
p_{\mathscr{B}}(f) \leqslant A p(f)
$$

The Dirichlet space, which consists of all analytic functions $f: D \rightarrow C$ with

$$
\int_{D}\left|f^{\prime}(z)\right|^{2} d \lambda(z)<\infty
$$

shows that $p$ need not be equivalent to $p_{\mathscr{B}}$. (Here $\lambda$ is Lebesgue area-measure on $D$ and $p$ is the square root of the above integral.)

Corollary 1. Let $(X, p)$ satisfy the hypotheses of the theorem. Then the kernel $p^{-1}(0)$ of the seminorm $p$ is contained in the constant functions.

Proof. The kernel of $p_{\mathscr{B}}$ is the constant functions.

COROLlaRY 2. Let $H(D)$ denote the linear space of analytic functions on $D$ equipped with the topology of uniform convergence on compact subsets of $D$. Then the only closed Möbius-invariant subspaces of $H(D)$ are $\{0\}, H(D)$, and the constant functions.

Proof. Let $E$ be a closed Möbius-invariant subspace of $H(D)$ with $E \neq$ $H(D)$. Then there exists a nonzero continuous linear functional $L: H(D) \rightarrow$ C with $L(f)=0$ for all $f \in E$. For $f \in H(D)$, set

$$
p(f)=\operatorname{Sup}\{|L(f \circ \phi)| \mid \phi \in \operatorname{Aut}(D)\} .
$$

Define $X$ to be $X=\{f \in H(D) \mid p(f)<\infty\}$.

Then $(X, p)$ satisfies the hypotheses of the theorem ( $L$ is a decent continuous linear functional on $(X, p))$. Thus, by Corollary $1, p^{-1}(0)$ is contained in the constant functions.

Note that $E \subseteq p^{-1}(0) \subseteq X$. Thus $E$ is contained in the constants. Therefore $E$ is either $\{0\}$ or the constant functions, as required.

The following lemma is the key to the proof of the main theorem.

LEMMA. For each $n \geqslant 1$, the seminorm $p_{n}$ defined on analytic functions $f$ : $D \rightarrow \mathrm{C} b y$

$$
p_{n}(f)=\operatorname{Sup}\left\{\left|(f \circ \phi)^{(n)}(0)\right| \mid \phi \in \operatorname{Aut}(D)\right\}
$$

is equivalent to the Bloch seminorm $p_{\mathscr{B}}$. 
Proof. The proof will be given for $n=3$. The general case is similar.

Consider $\phi_{a}(z)=(z+a) /(1+\bar{a} z)$, where $a \in D$. Then

$$
\begin{aligned}
\left(f \circ \phi_{a}\right)^{(3)}(0)= & f^{(3)}(a)\left(1-|a|^{2}\right)^{3}-6 f^{\prime \prime}(a)\left(1-|a|^{2}\right)^{2} \bar{a} \\
& +6 f^{\prime}(a)\left(1-|a|^{2}\right) \bar{a}^{2} .
\end{aligned}
$$

Now take $a=(1 / 2) e^{i \theta}$ and observe that

$$
(1 / 2 \pi) \int_{\Theta=0}^{2 \pi} a^{2}\left(f \circ \phi_{a}\right)^{(3)}(0) d \Theta=(9 / 32) f^{\prime}(0)
$$

A simple estimate yields

$$
\left|f^{\prime}(0)\right|<(8 / 9) p_{3}(f)
$$

Applying this last inequality to $f \circ \phi$ in place of $f$ and using $p(f \circ \phi)=p(f)$, we obtain

$$
p_{1}(f)=\operatorname{Sup}\left\{\left|(f \circ \phi)^{\prime}(0)\right| \mid \phi \in \operatorname{Aut}(D)\right\}<(8 / 9) p_{3}(f) .
$$

But $p_{1}=p_{\mathscr{B}}$, as can be verified easily by using the fact that each $\phi \in$ $\operatorname{Aut}(D)$ has the form $\phi(z)=e^{i \lambda}(z+a) /(1+\bar{a} z)$ for some $\lambda \in \mathbf{R}, a \in D$.

Thus $p_{3}$ dominates $p_{\mathscr{B}}$. It is not hard to show that $p_{\mathscr{B}}$ dominates $p_{3}$ by using the Cauchy integral formula to estimate $f^{\prime \prime \prime}(z)$ and $f^{\prime \prime}(z)$.

Proof of Theorem. Let $(X, p)$ satisfy the hypotheses of the theorem. Let $L: X \rightarrow \mathrm{C}$ be a nonzero linear functional on $X$ such that

$$
|L(f)| \leqslant p(f)
$$

and

$$
|L(f)| \leqslant A \operatorname{Sup}\{|f(z)| \mid z \in K\}
$$

for all $f \in X$, where $K$ is a compact subset of $D$ and $A>0$.

Then $L$ extends to a continuous linear functional (also called $L$ ) on $H(D)$ which satisfies (2) for each $f \in H(D)$ (by the Hahn-Banach Theorem).

Now, by [3], $L$ has a representation

$$
L(f)=(1 / 2 \pi i) \int_{|z|=r} f(z) g(z) d z / z
$$

for some function $g(z)$ analytic in a neighborhood of $\{z \in \mathbf{C}|| z \mid>r\}$ and some $0<r<1$. Also, it can be supposed that the Laurent series for $g$ about the origin has the form $g(z)=\sum_{n=N}^{\infty} b_{n} z^{-n}$, where $b_{N} \neq 0$ and $N>0$.

Recall inequality (1) now. Take $\phi(z)=e^{i \lambda} z(\lambda \in \mathbf{R})$ and apply (1) to $f \circ \phi$. Then a change of variables yields

$$
\left|(1 / 2 \pi i) \int_{|z|=r} f(z) g\left(e^{i \lambda} z\right) d z / z\right| \leqslant p(f \circ \phi)=p(f) .
$$

Next observe that

$$
(1 / 2 \pi) \int_{\lambda=0}^{2 \pi} e^{i N \lambda}\left[(1 / 2 \pi i) \int_{|z|=r} f(z) g\left(e^{i \lambda} z\right) d z / z\right] d \lambda=b_{N} f^{(N)}(0),
$$


an equation which can be checked by using Fubini's theorem. Thus (3) implies

$$
\left|f^{(N)}(0)\right| \leqslant\left(1 /\left|b_{N}\right|\right) p(f) .
$$

Apply (4) to $f \circ \phi$ in place of $f$ and take a supremum to get

$$
p_{N}(f)=\operatorname{Sup}\left\{\left|(f \circ \phi)^{(N)}(0)\right| \mid \phi \in \operatorname{Aut}(D)\right\}<\left(1 /\left|b_{N}\right|\right) p(f) .
$$

If $N>0$, then the Lemma yields the desired conclusion.

If $N=0$, then (5) says that

$$
p_{0}(f)=\operatorname{Sup}\{|f(z)| \mid z \in D\}=\|f\|_{\infty} \leqslant\left(1 /\left|b_{0}\right|\right) p(f) .
$$

Thus, in the case $N=0, X$ is contained in $H^{\infty}$, the bounded functions, and $p$ dominates the supremum norm $\|\cdot\|_{\infty}$. Since the supremum norm dominates $p_{\mathscr{B}}$ and $H^{\infty} \subseteq \mathscr{B}$ (see [1]), this is a stronger conclusion than the desired one.

To guarantee this stronger conclusion, it is sufficient to suppose $1 \in X$ and $L(1) \neq 0$, where 1 denotes the constant function 1 .

Here is an example which shows that the hypothesis in our theorem, that there exist a decent linear functional on $X$, cannot be dropped.

EXAMPLE 1. Let $\Omega$ be the family of all paths $\gamma$ in $D$ that tend nontangentially to a point on $\partial D$. Let $E$ be a set of angular measure zero on $\partial D$ and let $N_{E}$ be the family of all paths in $\Omega$ that have their endpoint in $E$. We call $N_{E}$ a "null set in $\Omega$." We define

$$
p(f)=\operatorname{ess} \sup \{\lim \sup |f(z)|:|z| \rightarrow 1, z \in \gamma: \gamma \in \Omega\},
$$

where "ess sup" denotes the infimum of the suprema over $\Omega \backslash N_{E}$, where $N_{E}$ ranges over all null sets in $\Omega$. It is easy to see that $p$ is a seminorm. (It follows from [2, p. 429] that $p$ is actually a norm.) Since Möbius transformations take paths in $\Omega$ into paths in $\Omega$, and sets of angular measure zero to sets of angular measure zero, we see that $p$ is Möbius-invariant, and so is the space $X$ of all holomorphic $f$ with $p(f)<\infty$. Now let $f(z)=\exp ((1+z) /(1-z))$. It is easy to see that $p(f)=1$ because $f$ has boundary values of modulus 1 at every point except $z=1$, but clearly $f$ is not a Bloch function.

Incidentally, this provides an example of a normed algebra of analytic functions in $D$ with no decent linear functionals. This algebra contains $H^{\infty}$ isometrically.

There is an example which shows that $p$ need not be equivalent to the supremum norm if the additional hypotheses mentioned above are assumed to hold.

EXAMPLE 2. Let $X$ be the set of all analytic functions $f$ on $D$ representable as a finite sum

$$
f(z)=\sum_{n=1}^{N} \alpha_{n}\left(z+a_{n}\right) /\left(1+\bar{a}_{n} z\right)
$$

where $\left(a_{n}\right)_{n}$ is a set of $N$ distinct elements of $D$ and $\left(\alpha_{n}\right)_{n}$ is a set of $N$ complex numbers, $N=0,1,2, \ldots$ Define a norm $p$ on $X$ by $p(f)=$ 
$\sum_{n=1}^{N}\left|\alpha_{n}\right|$, where $f(z)$ has the form (6). The norm $p$ is well defined because the Blaschke factors $(z+a) /(1+\bar{a} z)(a \in D)$ are linearly independent. This can be seen by continuing the factors to the Riemann sphere and considering poles.

Now take $L(f)=f(0)$. Then $(X, p)$ and $L$ satisfy the hypotheses of the theorem and the additional hypotheses mentioned above. However, $p$ is not equivalent to the supremum norm, as the sequence

$$
f_{n}(z)=z-(z-1 / n) /(1-(1 / n) z)
$$

shows.

Finally, it is an open question whether or not the theorem holds in the unit ball in $\mathbf{C}^{m}$. Bloch functions on bounded symmetric domains in $\mathbf{C}^{m}$ were studied in [5]. It surely does not hold in the polydisc $D^{m}$ in $\mathbf{C}^{m},(m>2)$, as the following example shows. We take $m=2$ for convenience.

EXAMPLE 3. Consider the seminorm

$$
p(f)=\sup \left\{\left|\frac{\partial^{2}}{\partial z \partial w}(f \circ \phi)(z, w)\right|_{z=0 ; w=0} \mid: \phi \in \operatorname{Aut}\left(D^{2}\right)\right\} .
$$

Here, $\operatorname{Aut}\left(D^{2}\right)$ consists of all biholomorphic maps of $D^{2}$ onto $D^{2}$. Every element $\phi$ of $\operatorname{Aut}\left(D^{2}\right)$ is (see [4, p. 167]) of the form

$$
\phi(z, w)=\left(\phi_{1}(z), \phi_{2}(w)\right) \quad \text { or } \phi(z, w)=\left(\phi_{1}(w), \phi_{2}(z)\right)
$$

where $\phi_{1}, \phi_{2} \in \operatorname{Aut}(D)$. Let $X$ be the space of all holomorphic functions $f$ on $D^{2}$ for which $p(f)<\infty$. It is easy to see that $(X, p)$ satisfies the hypotheses of the theorem (when stated for $D^{2}$ ). Any Bloch function $f$ on $D^{2}$ must satisfy (see [5])

$$
\sup \left\{\left|\frac{\partial f}{\partial z}(z, w)\right|\left(1-|z|^{2}\right):(z, w) \in D^{2}\right\}<\infty .
$$

Now take $f(z, w)=(1-z)^{-1}$ so that $p(f)=0$ but (7) fails. It may well be that this space $(X, p)$ is the largest decent space of holomorphic functions in $D^{2}$ with a seminorm that satisfies $p(f \circ \phi)=p(f)$ for all $\phi \in \operatorname{Aut}\left(D^{2}\right)$. We hope to investigate this question in the future.

\section{REFERENCES}

1. J. M. Anderson, J. G. Clunie and Ch. Pommerenke, On Bloch functions and normal functions, J. Reine Angew. Math. 270 (1974), 12-37.

2. G. M. Goluzin, Geometric theory of functions of a complex variable, Transl. Math. Monos., vol. 26, Amer. Math. Soc., Providence, R. I., 1969.

3. L. A. Rubel and B. A. Taylor, Functional analysis proofs of some theorems in function theory, Amer. Math. Monthly 76 (1969), 483-489.

4. W. Rudin, Function theory in polydiscs, Benjamin, New York, 1969.

5. R. M. Timoney, Bloch functions in several complex variables, Thesis, Univ. of Illinois at Urbana-Champaign, 1978.

Departmant of Mathematics, University of Illinois at Urbana-Champaign, Urbana, ILLINOIS 61801

Department of Mathematics, Indiana University, Bloommaton, Indinn 47401 